\title{
Computational Study and Analysis of the Kinetic Isotope Effects of the Rearrangement of cis-Bicyclo[4.2.0]oct-7-ene to cis,cis-Cycloocta-1,3-diene
}

Carlos Silva López, Olalla Nieto Faza and Ángel R. de Lera ${ }^{\dagger}$

February 15, 2006

Departamento de Química Orgánica, Facultade de Química, Universidade de Vigo, Lagoas Marcosende, E-36310, Vigo, Galicia, Spain.

${ }^{\dagger}$ Correspondence: qolera@uvigo.es, fax: 34986811940 


\section{B3LYP/6-31G(d) Cartesian Coordinates and SCF Energies}




\section{1}

SCF Energy: -312.020603619

C 0.4697910 .6501630 .792247

C $0.4697910 .650163-0.792247$

C $-0.366226-0.489150-1.386047$

C $0.068191-1.839805-0.780278$

C $0.068191-1.8398050 .780278$

C $-0.366226-0.4891501 .386047$

H 1.4823560 .6346651 .220578

H - $0.268434-0.5184762 .478850$

H - $1.428544-0.3115561 .175824$

H - $0.586168-2.6333021 .160477$

H $1.075124-2.0775491 .147084$

H - $0.586168-2.633302-1.160477$

H $1.075124-2.077549-1.147084$

H - $1.428544-0.311556-1.175824$

H - $0.268434-0.518476-2.478850$

C $-0.1408232 .036706-0.670475$

H $1.4823560 .634665-1.220578$

H - 0.459928 2.758735 - 1.419309

C -0.1408232 .0367060 .670475$

H - 0.4599282 .7587351 .419309

\section{2-conrot}

SCF Energy: -311.962680468

C 1.0024081 .0334000 .470104

C 2.1496340 .2829410 .085772

C $1.672411-0.950364-0.329575$

C $0.427940-1.0959380 .341804$

C - $0.909163-1.476544-0.216272$

C - $1.913401-0.4026120 .290441$

C - $1.5859581 .066703-0.083156$

C - $-0.1239331 .372755-0.500824$

H 1.0785451 .7483811 .301460

H 3.1834440 .6108430 .205822

H $2.059231-1.616588-1.100573$

H $0.447010-1.0120081 .425314$

H $-0.887549-1.515431-1.312064$

H - $1.254380-2.4578490 .143332$

H - $1.950026-0.5000891 .382953$

H - $2.918298-0.652847-0.070183$

H - 1.8649651 .7146120 .757708

H - $2.2223241 .379788-0.921345$

H $0.0861770 .865396-1.448066$

H - $0.0764932 .453745-0.714117$ 


\section{3}

SCF Energy: -312.010620752

C - $1.867734-0.8502400 .115689$

C $-0.564294-1.4694570 .381334$

C - $1.8985410 .459790-0.196271$

C $0.430766-1.253621-0.492193$

C - $0.7592521 .478103-0.252034$

C $1.822441-0.904779-0.086739$

C 0.5602441 .3095920 .574131

C $1.8029960 .656032-0.092580$

H - $2.803699-1.3983270 .227722$

H - $0.349838-1.8475891 .383803$

H - $2.8748320 .903256-0.403507$

H $0.148548-0.915426-1.490756$

H -0.496105 $1.664875-1.305646$

H - 1.2104672 .4245020 .078798

H $2.043417-1.2734840 .922792$

H $2.607006-1.268277-0.762639$

H 0.8658482 .3277210 .849795

H 0.3355990 .8004111 .519113

H 2.7085271 .0272210 .406197

H $1.8662471 .002600-1.133690$

\section{4-dbi}

SCF Energy: -311.959757679

C $1.946800-0.326442-0.277652$

C $1.160867-1.461857-0.072842$

C $1.6143820 .997355-0.013665$

C - $0.159089-1.4739720 .559394$

C 0.3033371 .5251250 .516589

C $-1.418929-1.205632-0.210008$

C $-0.9348641 .356906-0.424454$

C - $1.9283120 .248164-0.032662$

H $2.922841-0.494035-0.734881$

H $1.547837-2.406545-0.466780$

H $2.3654311 .744667-0.262521$

H - $0.224886-1.4451041 .649433$

H 0.0699291 .0678571 .487934

H 0.4374312 .5937420 .720668

H - $1.249339-1.397279-1.277617$

H - $2.223220-1.8837490 .114970$

H - $1.4894652 .303980-0.430077$

H - $0.5923531 .200224-1.455311$

H - $2.8434520 .364650-0.629961$

H -2.225905 0.3937131 .015948 


\section{5}

SCF Energy: -312.030993361

C $0.6985281 .630158-0.237047$

C -0.6987441 .6300850 .237068$

C -1.7096870 .7415220 .253153$

C - $1.817106-0.691832-0.194929$

C $-0.519852-1.410261-0.572775$

C $0.520054-1.4101710 .572839$

C $1.817197-0.6916360 .194878$

C $1.7095710 .741712-0.253170$

H $0.9783302 .621707-0.598934$

H - 0.9786632 .6215980 .598963

H -2.649687 1.1265110 .652139

H -2.310603 -1.252484 0.615587

H - $2.527433-0.735048-1.036296$

H - $0.769657-2.439088-0.859737$

H - $0.091398-0.940803-1.466059$

H $0.091593-0.9406951 .466108$

H $0.769973-2.4389550 .859857$

H $2.527626-0.7348001 .036157$

H $2.310644-1.252228-0.615710$

H $2.6495111 .126824-0.652176$

\section{2-disrot}

SCF Energy: -311.933299109

C 0.2563711 .9272590 .669231

C -0.3244120 .7480761 .344143$

C $0.383863-0.5671081 .491320$

C $-0.324412-1.7514550 .777560$

C $0.2563711 .927259-0.669231$

C $-0.3244120 .748076-1.344143$

C $0.383863-0.567108-1.491320$

C $-0.324412-1.751455-0.777560$

H 0.6492052 .7678341 .250268

H $0.6492052 .767834-1.250268$

H - 1.2575250 .8724201 .896471

H - $1.2575250 .872420-1.896471$

H $0.447813-0.8363082 .558682$

H $0.447813-0.836308-2.558682$

H $1.416773-0.4796331 .135873$

H $1.416773-0.479633-1.135873$

H - $1.357027-1.7898411 .145100$

H - $1.357027-1.789841-1.145100$

H $0.152304-2.6751001 .130391$

H $0.152304-2.675100$-1.130391 
4-[1,5]-H

SCF Energy: -311.921520292

C $-1.403520-1.356852-0.015269$

C $-2.176283-0.1977920 .134157$

C -1.5711811 .0832980 .001125$

C - $0.2013991 .379174-0.320136$

C 1.1970061 .2425880 .386488

C - $0.029057-1.0136270 .151966$

C $2.0870270 .068713-0.163896$

C $1.420711-1.368444-0.076999$

$\mathrm{H}-1.811217-2.268125-0.446893$

H -3.262104 -0.244212 0.102439

H - $2.2760411 .887117-0.213764$

H -0.148923 2.223249-1.020595

H -0.065981 -0.028942 -0.665179

H - $0.007117-0.4313421 .069228$

H 1.0913651 .1727031 .475260

H 1.7446372 .1742140 .202425

H 3.0545540 .0789130 .350938

H $2.2899180 .287584-1.220101$

H $1.616404-1.942852-0.988384$

H $1.834684-1.9306530 .770005$

CASSCF $(4,4) / 6-31 G(d)$ Cartesian Coordinates and SCF Energies 


\section{1}

MCSCF Energy: -309.920319690

C $0.0085079556-0.0051253172-0.6715557395$

C $0.0085079556-0.00512531720 .6715557395$

C $1.51874129400 .0012906910-0.8029342832$

C 1.51874129400 .00129069100 .8029342832

C $2.2209469184-1.2214664998-1.3875991090$

C $2.2209469184-1.22146649981 .3875991090$

C $3.6233081120-1.3700100298-0.7774105840$

C $3.6233081120-1.37001002980 .7774105840$

H - $0.7741832901-0.0078831710-1.4093435603$

H - $0.7741832901-0.00788317101 .4093435603$

H $1.93030153770 .9138980130-1.2268555267$

H 1.93030153770 .91389801301 .2268555267

H $1.6348187434-2.1132875818-1.1843232472$

H $1.6348187434-2.11328758181 .1843232472$

H $2.2955600210-1.1436976041-2.4691465282$

H $2.2955600210-1.14369760412 .4691465282$

H 4.0802216452 -2.2817204595 - 1.1500627337

H $4.0802216452-2.28172045951 .1500627337$

H $4.2444800628-0.5546580409-1.1385076783$

H 4.2444800628 - 0.55465804091 .1385076783

\section{2-conrot}

MCSCF Energy: -309.855116380

C - $1.6407117389-0.92817512030 .3101058610$

C - $2.09562723850 .3145645706-0.0737252541$

C $-0.3867957456-1.1202897871-0.3547145565$

C $-0.95450894421 .0838364065-0.4804268776$

C $0.9443676944-1.47514858000 .2371232274$

C 0.18178085101 .38888811460 .4906298534

C $1.9481720277-0.4213000667-0.2796486031$

C 1.62530225031 .05602474730 .0600486875

H - $2.0631594665-1.59627082331 .0419418726$

H -3.1151498698 $0.6629407660-0.1149524451$

H - $0.4019445652-1.0514044867-1.4265162728$

H - $1.06231149631 .8170731786-1.2721049211$

H $0.9013027755-1.47724391121 .3223589938$

H - 0.02714956660 .88604808941 .4272109776

H $1.2879641186-2.4595894452-0.0755697017$

H 0.15885780142 .45607141340 .7126601419

H $2.9410873447-0.65845572220 .0909624268$

H 2.2701190989 1.37954406970 .8732006664

H $1.9967631683-0.5372551740-1.3586380378$

H $1.89511049401 .6744376649-0.7918845533$ 


\section{3}

MCSCF Energy: -309.917375676

C $1.8547905122-0.7951804520-0.1162208703$

C $0.5487287668-1.4298653718-0.3844926640$

C 1.87591055400 .52162399180 .1700846641

C $-0.4492267931-1.26028583310 .5010453955$

C 0.71030509971 .50793978810 .2708241578

C $-1.8312036586-0.88213346220 .0767792700$

C $-0.59228052351 .3399730031-0.5721438872$

C -1.82620102930 .66751538820 .0826472027$

H $2.7807351680-1.3379606201-0.2103633224$

H $0.3227104473-1.7088342136-1.4032450671$

H 2.83649577690 .97648807260 .3578417994

H - $0.1803419581-0.95535876751 .5010316401$

H 0.44089966711 .62075220541 .3198413757

H $1.13049288072 .4705072537-0.0079888692$

H -2.0401174867 -1.2496175690 -0.9239196099

H - $2.6128721979-1.25005447980 .7350156049$

H $-0.90577947252 .3482039728-0.8289868581$

H - $0.36296512980 .8562542686-1.5165868631$

H - $2.72003154921 .0259100147-0.4219447673$

H - 1.90387642681 .01329418601 .1114040059

\section{4-dbi}

MCSCF Energy: -309.858988005

C - $1.90653047060 .3090802373-0.2967246387$

C $-1.14869662421 .4559859342-0.0720905044$

C - $1.5610559092-1.0186740383-0.0376730720$

C 0.18993546771 .49305754230 .5522488146

C $-0.2575152715-1.54394186660 .5216227032$

C $1.43506712081 .1818027911-0.2333792520$

C $0.9868346934-1.3821274465-0.3933576157$

C $1.9529367912-0.2502149241-0.0174236841$

H - $2.87100605110 .4614689337-0.7544278199$

H - $1.55297442002 .3909538674-0.4267999331$

H - 2.2898943419-1.7619370121 -0.3100199282

H 0.25264468541 .40983424101 .6257909873

H - $0.0538253265-1.09913156971 .4913173595$

H $-0.3999084090-2.60226324660 .7139268028$

H $1.24589308541 .3408378730-1.2917339181$

H 2.22871948751 .87120708500 .0495032875

H $1.5532182551-2.3085876311-0.3577056018$ H $0.6690636020-1.2642651688-1.4259514516$ H $2.8637316897-0.3706544678-0.5997021339$ H 2.2425510763 -0.3679590075 1.0254011095 


\section{5}

MCSCF Energy: -309.937884505

C $0.6855962662-1.59466165120 .2847762294$

C $-0.6858221554-1.5945681045-0.2847315766$

C $-1.6962055487-0.7050595788-0.2965768264$

C -1.80964759370 .70757796490 .2222272492$

C -0.51168950701 .42997169510 .5771834788$

C $0.51189890411 .4298901987-0.5771890609$

C $1.80975296310 .7073131597-0.2222254964$

C $1.6961081074-0.70529884860 .2966035124$

H $0.9271919527-2.55351950050 .7173745823$

H $-0.9275551553-2.5533986823-0.7173137040$

H - $2.6075354722-1.0589011312-0.7531511413$

H -2.3293575420 $1.2861715593-0.5401619863$

H -2.4743024303 0.70439556611 .0850345207

H -0.76348146142 .45008820140 .8520375835$

$\mathrm{H}-0.07502479800 .98084500151 .4626144337$

H $0.07516836920 .9808158834-1.4626140852$

H $0.76383846462 .4499667723-0.8520560667$

H $2.47440324270 .7040191859-1.0850356024$

H 2.32955059671 .28584406340 .5401512892

H 2.6073879866 - 1.05926367290 .7531823579

\section{2-disrot}

MCSCF Energy: -309.838563497

C $1.88156889540 .0466510008-0.6714863176$

C 1.88156889540 .04665100080 .6714863176

C $0.6743813468-0.3801043537-1.4161991333$

C $0.6743813468-0.38010435371 .4161991333$

C $-0.58529092990 .4422206617-1.5023317780$

C -0.58529092990 .44222066171 .5023317780$

C $-1.7999533890-0.1721681754-0.7768840011$

C $-1.7999533890-0.17216817540 .7768840011$

H $2.76135534330 .3435122926-1.2233019296$

H 2.76135534330 .34351229261 .2233019296

H $0.7692598232-1.2101895545-2.0968842021$

H $0.7692598232-1.21018955452 .0968842021$

H - $0.86317936110 .5509484867-2.5494246747$

H -0.86317936110 .55094848672 .5494246747$

H - $0.39988982351 .4463227050-1.1356747210$

H -0.39988982351 .44632270501 .1356747210$

H - $1.9208865085-1.1897565650-1.1362067273$

H - $1.9208865085-1.18975656501 .1362067273$

H -2.6812419843 $0.3653731155-1.1157960344$

H -2.6812419843 0.36537311551 .1157960344 


\section{4-[1,5]-H}

MCSCF Energy: -309.809357892

C $1.3622823804-1.35361767400 .0287578342$

C $2.1289182985-0.1813453534-0.1271196551$

C $1.52530841801 .0933696042-0.0109865743$

C 0.15028229931 .38632485690 .3485344622

C $-1.22925256871 .2447737555-0.3782491885$

C $-0.0039513808-1.0031876494-0.1345486288$

C -2.11580770020 .06293466350 .1472941680$

C $-1.4529328985-1.36530621090 .0638799548$

H $1.7651703814-2.24007205240 .4854084052$

H $3.2041081927-0.2212542976-0.1034021364$

H 2.21974178581 .90007821970 .1609494541

H 0.11026662112 .23853072511 .0190279606

H $0.0169216614-0.01400777020 .7576720115$

H - $0.0157287773-0.4052520415-1.0208344535$

H - $1.11050849441 .1880149379-1.4549824145$

H - $1.78518560382 .1587588025-0.1946947333$

H -3.0647820289 $0.0755424691-0.3797709674$

H - 2.33679423400 .27333451971 .1900464323

H - $1.6610405126-1.93733026450 .9604476400$

H - $1.8423132166-1.9203245037-0.7846089660$ 


\section{$\operatorname{CCSD}(T) / 6-31 G(d)$ energies}

\begin{tabular}{lr} 
molecule & Energy \\
\hline $\mathbf{1}$ & -311.023178 \\
$\mathbf{2}$-conrot & -310.962481 \\
$\mathbf{3}$ & -311.011630 \\
$\mathbf{4}$ & -310.942826 \\
$\mathbf{5}$ & -311.028132 \\
$\mathbf{2 - d i s r o t}$ & -310.927762 \\
\hline
\end{tabular}

MRMP(4,4)/6-31G(d) energies

\begin{tabular}{lr} 
molecule & Energy \\
\hline $\mathbf{1}$ & -310.911901 \\
$\mathbf{2 - c o n r o t}$ & -310.860433 \\
$\mathbf{3}$ & -310.905369 \\
$\mathbf{4}$ & -310.846923 \\
$\mathbf{5}$ & -310.921676 \\
2-disrot & -310.825245 \\
\hline
\end{tabular}

